\title{
Agôn
}

Revue des arts de la scène

7| 2015

La Distribution

\section{Pratiques, pensées et politiques de la distribution}

\section{Barbara Métais-Chastanier, Anne Pellois et Julie Sermon}

\section{(2) OpenEdition \\ Journals}

Édition électronique

URL : http://journals.openedition.org/agon/3476

DOI : 10.4000/agon.3476

ISSN : 1961-8581

Éditeur

Association Agôn

\section{Référence électronique}

Barbara Métais-Chastanier, Anne Pellois et Julie Sermon, « Pratiques, pensées et politiques de la distribution », Agôn [En ligne], 7 | 2015, mis en ligne le 06 novembre 2015, consulté le 03 mai 2019. URL : http://journals.openedition.org/agon/3476 ; DOI : 10.4000/agon.3476

Ce document a été généré automatiquement le 3 mai 2019.

Association Agôn et les auteurs des articles 


\title{
Pratiques, pensées et politiques de la distribution
}

\author{
Barbara Métais-Chastanier, Anne Pellois et Julie Sermon
}

«C'est ainsi que ma science prévoit

Que tout vivant joue son rôle :

La justice distributive,

C'est moi ; voici ce qui vous convient. "

Calderón, Le Grand Théâtre du Monde.

1 Au début des années 1980, une enquête menée auprès de metteurs en scène pose la question des modalités, concrètes et symboliques, de la distribution ${ }^{1}$. Mise à part cette enquête, exclusivement ancrée dans le champ théâtral, aucune étude systématique n'a été consacrée à la distribution dans les arts de la scène, alors même que celle-ci engage et articule des questionnements décisifs, au croisement de l'artistique, du politique et de l'économique.

2 L'apparente simplicité avec laquelle on définit la distribution - répartition entre les interprètes des rôles ou partitions d'une œuvre (théâtrale, chorégraphique, performative...) - ne doit en effet pas occulter les questions qui s'y embusquent et qui méritent d'être posées. En fonction de quels critères et de quels principes s'effectue cette opération de partage des rôles? Quel en est l'agent responsable? De quelle manière la distribution interagit-elle avec la création et/ou la réception d'une œuvre? En quoi détermine-t-elle des rapports de force ou de dépendance, politiques, symboliques et économiques, opérant aussi bien au sein des équipes de création (entre l'interprète et le/ la maître/sse d'œuvre, entre les interprètes eux/elles-mêmes) que vis-à-vis des contextes dans lesquels les œuvres s'inscrivent?

3 Opération à la fois discrète (puisqu'elle a lieu dans les amonts de la création) et manifeste (elle s'impose dans toute sa visibilité au public), la distribution est ce geste qui conditionne l'existence et détermine l'œuvre de part en part : ses processus de création, sa dramaturgie, son économie, son inscription dans l'espace social et dans le champ des 
imaginaires collectifs, qu'elle contribue tour à tour à conforter, subvertir, déconstruire voire enrichir. Loin de n'être qu'un geste circonscrit au champ artistique, la distribution s'articule en effet toujours à un principe ordonnateur (consensuel ou dissensuel, majoritaire ou minoritaire, théorique ou pragmatique) qui la déborde et qu'il importe de mettre au jour, car c'est à travers lui que s'actualise la dimension idéologique et donc politique de ces constructions symboliques que sont les œuvres.

En décidant de consacrer son dossier $n^{\circ} 7$ à la question de la distribution, la revue Agôn a donc eu pour volonté d'attirer l'attention des chercheur.e.s et d'inviter les artistes à s'exprimer sur cet impensé des arts vivants, et pour ambition de tenter de circonscrire un champ dont l'acuité - et l'actualité - des problématiques qu'il soulève (qu'on pense, dernièrement, à Exhibit $B$ de Brett Bailey ${ }^{2}$ ou à l'Othello blanc de Luc Bondy ${ }^{3}$ ) n'ont finalement d'égal que la difficulté à les saisir dans leurs différentes dimensions et conséquences. Dans cette introduction, nous nous proposerons d'ouvrir à la complexité de questions qui ne manqueront pas de trouver des réponses (circonstanciées) et des prolongements (singuliers) dans la lecture des articles qui constituent ce dossier.

\section{Distribution, rôles et processus de création}

5 Avant de désigner, par métonymie, l'ensemble des interprètes prenant part à la réalisation d'une œuvre (ainsi en est-il lorsque les critiques saluent une "belle distribution" ou regrettent une "distribution inégale"), le terme de distribution renvoie, ou bien à un geste de répartition d'un tout constitué (l'œuvre à représenter) entre les différentes parties (les interprètes), ou bien, dans les cas où l'œuvre ne s'élabore qu'au fil du travail des répétitions, au processus de détermination du rôle et de la place que chacun des membres de l'équipe de création sera amené à endosser. La première série de questions que pose la distribution est donc celle de la relation et des dialectiques spécifiques qui s'instaurent entre l'interprète et la "partie" qu'il prend en charge, que celle-ci lui préexiste, évolue ou s'invente avec lui.

Dans le cas où la distribution constitue une opération intermédiaire (entre une œuvre préexistante et sa concrétisation scénique), le choix des interprètes sera en priorité conditionné par les nécessités et les logiques de l'œuvre à représenter (nombre de personnages, type et genre des rôles, registre dramatique....). Ce principe général étant posé, demeure toutefois entière la question de savoir pourquoi et comment, selon les époques et les contextes, l'on décide d'attribuer un rôle fictif, forcément abstrait, à un interprète singulier qui l'incarnera effectivement. Qu'est-ce qui détermine, dans sa personne ou son parcours singulier, le fait qu'à un moment donné, il parait intéressant, approprié ou au contraire inapproprié de lui confier (ou qu'ille ${ }^{4}$ 'attribue) tel ou tel type de rôle? Qui statue sur le caractère compatible ou incompatible, pertinent ou inadéquat, de tel ou tel choix de distribution - le/la maitre/sse d'œuvre, l'interprète lui/elle-même, le répertoire, l'institution théâtrale, la société ? À quels présupposés (morphologiques et idéologiques), attentes (techniques) et règles (dramaturgiques) s'adossent le système conventionnel des "emplois" ou celui, en miroir inversé, du "contreemploi" ? Et du même coup : dans quelles mesure la distribution et les variations qu'elle autorise (selon les contextes : jeu sur la physionomie, l'âge, le sexe, la race...) engagent-elles les pratiques scéniques vers la confirmation d'une norme, d'un ordre ou d'une structure préalables (l'imaginaire collectif attaché à une œuvre, un rôle, une fonction, une identité...), ou, au contraire, contribuent-elles à déstabiliser, déconstruire, renouveler, non seulement la 
lecture des rôles et la réception des œuvres, mais aussi, celle des représentations en vigueur dans l'espace social?

7 Ainsi conçue comme opération intermédiaire, la distribution est en effet inséparable de la notion de "lecture" (celle qu'on produit d'un texte préexistant, et qu'on décide de partager avec une assemblée de spectateurs). À ce titre, elle renvoie à un régime d'organisation du travail très spécifique qui, à l'échelle millénaire de l'histoire du théâtre, ne concerne finalement qu'un tout petit pan de la production théâtrale: celle qui, à compter du XIX ${ }^{e}$ siècle, s'est fondée sur une séparation progressive des fonctions (auteurs, interprètes, metteurs en scène) - cette spécialisation, qui passe par l'affirmation et la définition des compétences, droits et devoirs propres à chacun.e, ayant pour notable conséquence d'instaurer une chronologie segmentée de la création (le temps de l'écriture est dissocié de celui du jeu et de la représentation). Dans ce système cloisonné, c'est alors

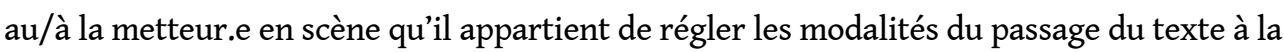
scène, du personnage à l'acteur.rice, et donc, à lui/elle que revient la responsabilité de rassembler et de composer une distribution à même de porter sa lecture du texte. C'est en effet en fonction des choix très concrets de distribution que ce/tte metteur.e en scène pourra faire, que sa lecture de l'œuvre sera estimée plus ou moins "fidèle", "originale" ou "iconoclaste", selon qu'ille a choisi de reconduire les conventions artistiques et les normes représentatives propres à cette œuvre et à son histoire, ou au contraire, de jouer des écarts et de créer des discordances avec cet horizon d'attente.

8 Si, pendant près d'un siècle (des années 1880 aux années 1980), c'est du même coup la figure du metteur en scène qui a cristallisé les attentions et les réflexions, et si c'est symptomatiquement à lui - et exclusivement à lui - que s'adressait le questionnaire sur la distribution que nous avons mentionné en introduction, il est significatif que, dans ce dossier, il soit relativement absent des réflexions, l'essentiel des contributions s'attachant à questionner des formes où la distribution, au lieu d'intervenir dans un second temps, en relation avec une œuvre déjà écrite, est au contraire une étape inaugurale du processus de création.

9 À l'instar des auteurs antiques ou classiques qui, comme le montrent les articles de Diomedes Gariazzo Lechini ${ }^{6}$ et de Céline Candiard ${ }^{7}$, écrivaient pour des acteur.trice.s aux compétences spécifiques, que les auteurs cherchaient précisément à exploiter ou à valoriser au mieux dans leur dramaturgie (ces considérations primant alors souvent sur celles de la construction de l'intrigue ou de la cohérence du caractère), la multiplication, ces dernières années, de formes scéniques nées d'écritures de plateau ou collectives, replace au cœur du geste artistique cette pratique de la distribution où l'on écrit pour - et compose avec - un ensemble d'interprètes (une troupe, un groupe) constitué en amont de l'œuvre. Dans leurs contributions, Stéphane Hervé ${ }^{8}$ et Esther Gouarné 9 s'attachent ainsi à analyser quels sont les endroits de porosité, les phénomènes d'influence et d'interférence entre la réalité de l'interprète et le(s) rôle(s) qu'il ou elle endosse, dans telle ou telle œuvre et d'une œuvre à une autre, cette approche comparative révélant du même coup le retour, à l'horizon de ces pratiques, d'un système d'emplois qui, pour être modernisé, n'en risque pas moins d'enfermer les interprètes dans un certain type de jeu et de représentation - sclérose que, chaque fois qu'elle s'est retrouvée en situation d'écrire pour des interprètes spécifiques, Noëlle Renaude ${ }^{1011}$ s'est pour sa part précisément attelée à éviter.

10 D'une autre manière, le recours à un procédé de distribution aléatoire - comme ont pu le faire Merce Cunningham ou, plus récemment, Gwenaël Morin - permet aux artistes de 
s'émanciper des logiques normatives de l'emploi / contre-emploi (dont les effets, conscients ou latents, sont toujours puissants), en même temps qu'il constitue une réponse, en forme d'échappée, à la prise de pouvoir décisionnaire qui est inhérente au geste de distribuer. On est toutefois en droit de penser que ces formes de distribution non intentionnelles ne sont pas, pour autant, exemptes de violence: si le hasard est un élément du processus démocratique, présupposant l'égale capacité de tous ${ }^{12}$ («Nous sommes tous égaux devant Molière » était-il d'ailleurs écrit au sommet de la carte de distribution des Molière de Gwenaël Morin), il implique aussi une soumission absolue de l'interprète à un principe abstrait, qui revient à troquer un arbitrage contre un arbitraire - la question de la relation particulière qu'ille peut nouer, d'une part, avec un rôle, et d'autre part, avec un.e chorégraphe ou un.e metteur.e en scène qui l'aurait choisi.e pour ses compétences particulières, se voyant du même coup frappée d'inanité.

Envisagée du point de vue de la construction des rôles et de son articulation aux processus de création, par exemple par le Collectif $\mathrm{X}^{13}$, la question de la distribution peut enfin, outre la forme paradoxale précédemment envisagée, être confrontée à deux cas de figure limites: quand les œuvres, textuelles ou scéniques, ne présentent a priori plus aucuns signes extérieurs de répartition (absence de personnages, voix anonymes non attribuées, disparition de rôles clairement définis), ou bien, dans le cadre de projets peu ou prou documentaires, quand il y a au contraire adéquation parfaite entre une subjectivité donnée et le rôle qu'elle endosse sur scène, autrement dit, quand le choix de ceux et celles avec qui l'on travaille est déjà geste d'écriture et mise en jeu du sujet (que serait Gardenia d'Alain Platel sans les sept anciens travestis réunis autour de Vanessa Van Durme? Madame Plaza sans les Aïtas choisies par Bouchra Ouizguen? Les projets de Rimini Protokoll sans ceux qu'ils nomment les « experts du quotidien »?). Dans ces deux cas, on peut alors s'interroger sur la pertinence qu'il y a à encore recourir à la notion de distribution - et c'est ce que font, chacun à leur manière, Violaine Chavane ${ }^{14}$ et Ismaël Jude $^{15}$, la première se proposant de parler, à propos du travail de Jérôme Bel, d'un "degré zéro de la distribution", le second revenant quant à lui sur le principe deleuzien d'une "distribution nomade".

\section{Distribution et modèles de représentation}

Dispositifs de représentation au sens esthétique, les arts de la scène, étudiés du point de vue de la distribution, le sont aussi au sens politique et social du terme: des figures de délégué sont appelées à représenter symboliquement le monde. Ce n'est donc pas seulement: me reconnais-je dans ces corps, dans ces voix, dans ces gestes? Mais bien plutôt : Est-ce cela que nous sommes ? Est-ce cela mon époque, mon pays, ma ville ? Sontce là mes contemporains ?

Sans aller jusqu'à rêver à l'improbable coïncidence d'une pure duplication démocratique de la scène et de la salle comme peut l'appeler de ses vœux Martin Crimp en exergue d' Atteinte à sa vie (pièce écrite pour "une troupe d'acteurs dont la composition devrait refléter la composition du monde au-delà du théâtre $\aleph^{16}$ ) ou d'une délégation statistique comme la réinvente Rimini Protokoll dans son dispositif des 100 pour cent appliqué aux différentes grandes villes du monde (Berlin, Zürich, Melbourne, Dresde, Cologne, Vienne, Londres, etc.), force est de reconnaître que la distribution s'articule toujours implicitement à des a priori concernant les notions d'identité et de société - d'un point de vue philosophique comme d'un point de vue politique et social. 

statisme, ou au contraire de labilité) que déploient les principes de distribution, autrement dit, questionner les liens qu'il est possible d'établir entre, d'un côté, le degré de caractérisation des rôles ou de structuration des emplois, et de l'autre, l'organisation politique et économique des sociétés dans lesquelles les œuvres s'inscrivent. De même, en effet, qu'on peut faire correspondre la structure rigide de la société classique et la distribution précise des emplois, de même, on peut se demander si le flottement des voix dans les dramaturgies contemporaines n'est pas à mettre en relation avec la volonté d'effacement de la question des classes dans les démocraties libérales. De manière plus circonstanciée, peuvent également être questionnés les phénomènes de transfert qui s'établissent, ou non, entre, d'un côté, le prestige et le rang social de l'acteur, et de l'autre, l'importance du rôle et le rang social du personnage qui lui est confié.

15

A l'intérieur de ces articulations entre distribution et représentation politique et sociale, il importe du même coup de se rendre particulièrement attentif aux phénomènes de circulations, de déplacements et de troubles que peuvent provoquer des distributions qui, parce qu'elles échappent à la dialectique emploi/contre-emploi, gagnent à être envisagées à l'aune des théories queer. En effet, si ce que les anciens appelaient " polytropie $~^{17}$, à savoir, la capacité de l'interprète à endosser plusieurs rôles, variant au gré des circonstances, est souvent le fruit d'une contrainte économique (impossibilité d'employer autant d'interprètes qu'il y a de rôles) ou structurelle (nécessité de faire jouer l'ensemble d'une troupe ou d'une promotion), elle ne se résume pas à ces impératifs logistiques : elle peut être délibérément choisie et explorée. Le travail d'un Jean-Marc Avocat, interprétant seul trois grands classiques de Racine (Bérénice, Phèdre et Andromaque ), celui d'Yves-Noël Genod notamment dans Manuel de liberté où se croisaient une Cerisaie et un Macbeth porté chacun par un.e seul.e comédien.ne, ou encore celui de Guillaume Baillard reprenant tous les rôles du Tartuffe de Molière dans la mise en scène de Gwenaël Morin, viennent troubler les catégories du rôle et avec elles, celle d'une binarité pensée autour de l'emploi. De même, les formes d'incarnation partagée (un même rôle pris en charge par plusieurs interprètes) ou de distributions variables (comme c'était le cas dans L'Avenir seulement, écrit et mis en scène par Mathieu Bertholet, ou dans la mise en scène de 11 septembre 2001, de Michel Vinaver par Robert Cantarella), peuvent se charger d'une fonction critique de mise à mal des conceptions essentialistes ou exclusives qui structurent l'espace social et les identités. En effet, par la tension qu'elles instaurent entre mise en ordre et dissémination, reprise et écart, ces choix interrogent et mettent au jour les effets de rencontre entre un corps et un rôle, entre une voix et une fonction travaillant ainsi à dénaturaliser et défaire ce qu'une distribution fixe peut malgré elle contribuer à immobiliser. C'est à ce trouble que s'intéressent diversement Matthieu Hocquemiller ${ }^{18}$, Camile Khoury ${ }^{19}$ et Marie Astier ${ }^{2021}$ : le premier en revenant, dans le cadre d'un entretien, sur les processus de distribution dans/de la performance postpornographique, la seconde en questionnant le principe de distribution à contregenre à travers l'étude de la figure du travesti dans le théâtre du XIX siècle, la troisième en s'intéressant à la manière dont la distribution de comédien.ne.s en situation de handicap chez Philippe Adrien participe à brouiller les frontières entre valides et nonvalides.

16 À l'inverse, la distribution peut venir conforter voire accentuer des catégorisations rigides, renforçant ainsi les rapports de domination qui structurent l'espace social. Tel est d'ailleurs le sens des critiques qui furent adressées à Brett Bailey, à propos d'Exhibit $B$, 
dont l'une des plus fécondes - celle de Dorcy Rugamba - pointait l'ambiguïté raciste qui présidait au choix de distribution : «Le spectacle devient le contraire de ce qu'il prétend faire. Peut-on dénoncer le racisme en reprenant des méthodes de profiling racial ? Cela pose des problèmes éthiques fondamentaux ${ }^{22}$.» Cette distribution racialisée qui reconduisait une vision stéréotypée de l'imaginaire colonial en distribuant des comédien.ne.s noir.e.s dans des tableaux confortant une vision misérabiliste n'a pas manqué de susciter des débats, rendus parfois inaudibles par le manque d'attention porté à leur spécificité. Trop rapidement rapprochés des discours de censure de l'extrême droite montés en épingle autour de Romeo Castellucci (Sur le concept du visage du fils de dieu) et de Rodrigo Garcia (Golgotha Picnic), ces critiques parfois maladroites en ce que certaines, notamment celles portées par la Brigade anti-négrophobie, en appelaient à l'interdiction de l'œuvre, n'en étaient pas moins légitimes: elles mettaient l'accent sur une distribution inégalitaire des visibilités que l'installation-performance venait renforcer - peut-être malgré elle. N’oublions pas Angela Davis qui rappelait que le roman La Case de l'oncle Tom, pourtant considéré par son autrice et son époque comme un ouvrage progressiste, n'en perpétuait pas moins « le racisme et le sexisme qui justifiait respectivement l'esclavage et l'exclusion des femmes de la bataille politique contre l'esclavage ${ }^{21}$. C'est à cet exercice de distinction franche et précise des intentions et des effets d'une œuvre que nous invite aussi ce dossier consacré à la distribution.

À l'inverse, le choix délibéré de Luc Bondy d'effacer la question raciale en distribuant Othello à un comédien blanc (Philippe Torreton), alors que la dramaturgie de la pièce de Shakespeare repose précisément sur la mobilisation d'un cadre historique et social qui place le personnage éponyme au centre d'un renversement politique, participe cette foisci d'un projet plus global d'invisibilisation des questions raciales, qui n'est que l'autre nom de l'impérialisme universaliste. En un sens, cette distribution (comme celle de Gérard Depardieu en Alexandre Dumas dans le film L'Autre Dumas réalisé par Safy Nebbou) vient confirmer un aveuglement par rapport au questions raciales et plus globalement par rapport aux dispositifs de domination, qu'ils soient de race, de genre ou de classe. En ce sens, les acteurs aveugles de la compagnie du $3^{\mathrm{e}}$ œil distribués par Philippe Adrien pour interroger les fonctions du voir, les interprètes du Theater HORA n'interprétant qu'eux-mêmes dans Disabled theater de Jérôme Bell, les acteurs d'origine nord-africaine ou africaine exigés par des auteurs tels Genet ou Koltès (voir notamment l'article d'Arnaud Maisetti ${ }^{23}$ ) pour l'interprétation de leurs personnages, ont pour effet d'interroger les rapports de force qui structurent la manière dont une société se donne elle-même à voir à travers ce(ux) qu'elle cache ou décide de la manière dont elle veut les voir. Elles interrogent les conditions du regard spectateur par des formes qui peuvent faire violence au principe voyeuriste du spectacle, tout autant qu'elles distribuent les possibles de ces mêmes visibilités : c'est d'ailleurs ce que dénonçait Georges Aminel, premier acteur noir à entrer au Français : «Je suis trop blanc, trop noir, le cheveu trop crépu ou pas assez. Bref, des amis qui me veulent du bien me demandent pourquoi je ne joue pas Othello mais jamais pourquoi je n'interprète pas Macbeth. C'est bien simple, j'ai passé mon temps à me barbouiller et à prendre un accent. Les faits sont là: j'ai débuté dans un rôle de Polynésien muet et depuis je ne compte pas les personnages de chamelier juif, brésilien ou arabe que j'ai endossés. Alors, si parce que mon père est Antillais, je dois toute ma vie incarner des Sud-Américains explosifs ou des Indigènes fanatiques, je préfère arrêter. » ( Le Figaro, 20/06/1979). Ce qui ainsi était mis en critique était moins l'invisibilisation que la réduction à des rôles simplistes, stéréotypés et de second plan. De ce point de vue, il serait d'ailleurs judicieux d'interroger ce qui sépare un personnage comme Othello d'un 
autre comme celui d'Aziz dans Le Retour au désert: dans quelle mesure l'un est-il plus complexe, plus affranchi, plus inédit dans l'espace politico-social - et donc plus critique que l'autre? Il serait également judicieux d'aller plus loin dans l'établissement des critères qui rendent un rôle ou un personnage "intéressant".

Le fait que nous n'ayons reçu pour ce dossier aucune proposition traitant des processus d'invisibilisation et de la violence des représentations (entendue au sens esthétique comme au sens politique et social) témoigne de cette difficulté conceptuelle et méthodologique à laquelle sont confronté.e.s bon nombre de chercheur.se.s en arts du spectacle. Car ce que révèle le peu d'écho que rencontrent ces questions, c'est une carence conceptuelle plus large qui ne manque pas d'en laisser plus d'un.e démuni.e face à ces questions : en effet, le peu de visibilité offerte, en France, aux approches postcoloniales, comme le manque d'intérêt pour des outils conceptuels développés notamment dans le cadre des Subaltern studies ou des Gender studies depuis plus de vingt ans en Inde et aux États-Unis, rend difficile une articulation intelligible des enjeux. Si l'on ajoute à cette non-réception française une tradition de l'artiste pantocrator indexant volontiers le registre de la création à celui de la subjectivité, occultant au passage la dimension collective et politique de ces constructions symboliques que sont les œuvres, on comprend que tout soit alors réuni pour favoriser une hégémonie culturelle qui, sous couvert de goût (qu'on imagine évidemment indéfinissable ou purement subjectif) et d'une croyance volontiers universaliste dans les pouvoirs de l'art, ne fait que dénier son appartenance à des systèmes de domination, qu'elle contribue par ailleurs à renforcer. La difficulté de l'association $\mathrm{H} / \mathrm{F}$ à obtenir des engagements concrets (programmation paritaire, politiques de distribution, etc.) de la part des directeur.rice.s de lieux ${ }^{24}$, la sous-représentation des interprètes métis, noirs, arabes ou asiatiques ou leur condamnation à des distributions stéréotypées ${ }^{25}$, le cantonnement des places décisionnaires à un mandarinat, sont autant de témoignages de cette confiscation opérée au service d'un empire artistique et culturel majoritairement parisien, masculin, blanc, bourgeois, hétérosexuel, cisgenre ${ }^{26}$ et validiste ${ }^{27}$. Il n'y a d'invisible qu'invisibilisé: distribuer, c'est dans tous les cas décider d'une certaine visibilité. Qui peut monter sur scène ? Pour faire quoi ? Selon quels critères et dans quelles conditions ? Que deviennent les acteurs-actrices et les danseursseuses vieillissants, pourquoi n'ont-ils plus droit à la même place s'interrogeait par exemple Thierry Baë dans son Journal d'inquiétude? Pourquoi les femmes sont-elles restées si longtemps interdites de scènes? N'était-ce pas parce que la société répétait à travers ses dispositifs esthétiques des principes d'empêchements politiques? Il est alors important de nommer explicitement la responsabilité de chacun.e dans le manque de diversité, qu'elle soit ethnique, culturelle, géographique ou sociale. La question de la distribution - entendue à la fois comme opération de répartition des rôles mais aussi comme outil de légitimation et de circulation entre des formes de culture considérées comme centrales et d'autres comme marginales - nous renvoie de manière à une posture de dénégation: difficile pour les chercheur.se.s comme pour les artistes (majoritairement parisien.nes, masculin, blanc.che.s, bourgeois.e.s, hétérosexuel.le.s, cisgenres et validistes), soucieux.ses bien souvent d'émancipation intellectuelle et poétique, si ce n'est politique, de reconnaître qu'illes participent, qu'illes le veuillent ou non, d'une distribution inégalitaire des privilèges qui, le plus souvent, leur bénéficie ${ }^{28}$. De ce point de vue, c'est à un véritable travail de déconstruction que nous invite ce dossier en nous plaçant au cœur de ces enjeux, manière peut-être d'ouvrir à une répartition véritablement égalitaire et multiculturelle des moyens de production comme des outils de création et de diffusion. 


\section{Distribution et rapports de production}

19

La distribution est un enjeu politique (qui a le pouvoir de distribuer?) et économique (quelle est la stratégie de (la) distribution à l'égard des spectateurs ?). La distribution fut d'abord l'apanage des directeurs de théâtre, des auteurs, voire des interprètes euxmêmes. Régie souvent par le système des emplois à partir du XVIII e siècle, propice à la mise en valeur des monstres sacrés à la fin du XIX ${ }^{e}$ siècle, sa subversion peut servir à frapper un grand coup médiatique. C'est par exemple ce que choisit de faire Victor Hugo lorsqu'il distribue en 1835 dans Angelo, tyran de Padoue, Mademoiselle Mars (comédienne excellant dans les rôles d'ingénue et d'amoureuse) dans le rôle de la courtisane Tisbé, et Marie Dorval (actrice de boulevard) dans celui de la noble Catarina, ajoutant au contreemploi le "choc étincelant des deux actrices" 28 qui se partagent le haut de l'affiche.

$\mathrm{Au} \mathrm{XX}^{\mathrm{e}}$ siècle, la distribution devient la prérogative du metteur en scène et du chorégraphe, choisissant soigneusement ses interprètes, avant que celle-ci soit remise en cause ces dernières années par un retour au pouvoir des directeurs de lieux d'une part, et par la prolifération des collectifs d'acteurs.trices effaçant la figure du metteur en scène d'autre part. Quel que soit le cas de figure, le distribution s'effectue non seulement selon des critères développés dans les deux parties précédentes, conscients (artistiques et dramaturgiques) ou inconscients (ressortissant de représentations plus ou moins assumées), mais également selon des critères économiques qui prennent en compte la viabilité d'un spectacle en terme de production, tournée, etc. Le spectacle des Gens d'Uterpan, Avis d'audition, analysé par Aline Jaulin ${ }^{29}$, rend compte de ces phénomènes de sélection propres à l'univers de la danse, et des contraintes économiques que ces choix suscitent.

Distribuer, c'est donc exercer très concrètement le pouvoir de décider qui l'on va employer, à quel prix, selon quelle importance. C'est par exemple orchestrer une œuvre au service d'une tête d'affiche qui dictera ses conditions et autour de laquelle s'organiseront la production et le processus de création, comme le développe Daniel Urrutiaguer ${ }^{30}$, ou travailler à la visibilité d'une structuration horizontale d'un collectif au point de souhaiter l'effacement des noms des interprètes ${ }^{31}$. Aussi différents soient-ils, ces choix impliquent des stratégies de production, de communication et de diffusion qui conditionneront l'horizon de l'œuvre et son inscription dans l'espace social. Les modalités de distribution peuvent par exemple induire un état de concurrence entre des interprètes aux compétences similaires. Pourquoi choisir un tel plutôt que tel autre, à compétences supposément égales, dans le cas de disciplines mettant en jeu des savoir-faire que l'on pense identifiables. Dans ce que Marion Guyez appelle "la grande famille du cirque" ${ }^{32}$, les mises en concurrence dans la dramaturgie du numéro des artistes est patente. Mais la distribution peut aussi se fonder sur un principe subvertissant d'une certaine manière la logique "marchande" et économique qui l'accompagne souvent. En travaillant sur le double principe des "guests" et de la disparition de l'interprète derrière une incarnation qui le dépasse absolument, Yves-Noël Genod, se qualifiant lui-même de "distributeur", travaille à critiquer les modalités de productions classiques (rangés sous la bannière de la "grande distribution") et les modèles verticaux de distribution qui l'accompagnent (liés notamment à une prépondérance du metteur en scène dans le geste de distribution dont il entend se démarquer) ${ }^{33}$. 

également voulu interroger le cas particulier des écoles et des promotions qui les constituent, en ce qu'elles reflètent, ou non, à la fois une certaine projection d'un état du marché du travail à la sortie de l'école et les modèles de représentations en vigueur dans ce paysage. Les critères de recrutement et de composition des promotions (essentiellement d'acteurs.trices ici) ont donc été examinées, afin de mettre au jour certains paramètres déterminants au moment du concours, mais aussi tout au long de la scolarité. Ce cas particulier qui consiste à sélectionner un groupe comme étant susceptible de contenir un maximum de distributions en puissance, a été envisagé du côté des institutions, à la fois dans une perspective historique, développée par Flore Augereau depuis la fondation du Conservatoire jusqu'à nos jours ${ }^{34}$, et par études de cas, via les entretiens de

rédéric Plazy pour la Manufacture à Lausanne ${ }^{35}$ et de Stanislas Nordey pour l'école du TNS à Strasbourg ${ }^{36}$. Le collectif $\mathrm{X}$, issu essentiellement mais pas exclusivement de la Promotion X de l'École de la Comédie de Saint-Étienne ${ }^{37}$, a quant à lui développé le point de vue des élèves: leur témoignage révèle des constantes clairement associées à des représentations collectives, lesquelles ne remettent pas du tout en cause les critères classiques et automatiques de distribution. L'exercice difficile du spectacle de sortie, évoqué par Noëlle Renaude du côté de l'écrivain à qui l'on passe commande, mais aussi par les $\mathrm{X}$ du côté des élèves confrontés au personnage parfois surprenant que l'on a écrit "pour eux" sans les connaître, laisse transparaitre les difficultés inhérentes à ces distributions a priori, avant l'écriture et pour des artistes qui doivent démontrer leur valeur.

Des critères de sélection du concours aux rapides distributions - rapides parce que soumises à la nécessité de l'efficacité - décidées par les intervenants dans les écoles, le constat n'est pas celui de l'originalité. Il aurait même tendance à renforcer les représentations rattachées au processus de distribution, estampillant dès l'école certains interprètes plus et/ou mieux "distribuables" que d'autres. Combien d'acteurs ou de danseurs seront-ils à jamais condamnés au second plan dès leur formation? Cette quasi absence de remise en cause des représentations, au nom souvent d'un impératif, très compréhensible, de débouché et d'efficacité de la formation, laisse peu de place à des modalités expérimentales de formation qui déplaceraient les apprenti.e.s comédiens.nes pour leur faire explorer d'autres voies que celles auxquelles illes semblent prédestiné.e.s dès leur entrée. Qui les formeraient en somme au théâtre tel qu'il pourrait être plutôt qu'au théâtre tel qu'il existe. Certes, se développent des dispositifs que l'on pourrait qualifier de "correctifs", visant à modifier les états de fait. Ainsi des dispositifs de classe préparatoire, mis en place au TNS et à l'École de la Comédie de Saint-Étienne ${ }^{38}$, visent à ouvrir les recrutements des concours à ce que l'on appelle "la diversité", cherchant ainsi à crever une forme de "plafond de verre" empêchant ces potentiels candidats de postuler, mais aussi à encourager une forme de discrimination positive en contradiction avec le paysage, largement blanc, constitué par l'ensemble des artistes d'aujourd'hui. Mais ces dispositifs ne font que souligner la persistance de schémas de représentation qui ne sont pas remis en cause, voire même pas relevés. 


\section{NOTES}

1. «Journal des créateurs. Comment je fais une distribution » in Revue L'Annuel du théâtre. Saison 1982-1983 (dir. Jean-Pierre Sarrazac, avec la collaboration de Pierre Ballet-Baz, Monette Berthommier, Bernard Faivre, Michelle Henry et Jean-Loup Rivière), L'Aire théâtrale / Les Fédérés, 1983, p. 9-35. Des extraits de cette enquête sont reproduits, avec l'aimable autorisation de Jean-Pierre Sarrazac, dans ce dossier : http://agon.enslyon.fr/index.php?id=3404

2. Exhibit B, installation-performance de Brett Bailey, présentée en décembre 2014 au TGP et en janvier 2015 au 104. Voir notamment l'article de Joseph Confavreux sur Mediapart qui présente une bonne synthèse des enjeux liés à la polémique : http://www.mediapart.fr/journal/cultureidees/021214/ce-que-dit-la-polemique-exhibit-b.

Voir aussi l'article d'Éric Fassin: http://blogs.mediapart.fr/blog/eric-fassin/291114/exhibit-brepresentation-duracisme-et-sous-representation-des-minorites-raciales

Voir enfin l'article d'Alice Carré dans Agôn pour un autre point de vue : http://agon.ens-lyon.fr/ index.php?id=3129

3. Othello, mise en scène de Luc Bondy. La pièce devait être présentée au Théâtre de l'Odéon à partir de janvier 2016. La mise en scène est reportée à l'année suivante en raison de l'état de santé de Luc Bondy. Voir notamment les articles suivants: Le Monde (16 oct. 2015) http:// abonnes.lemonde.fr/afrique/article/2015/10/16/pas-de-noirssur-scene-le-theatre-francais-estil-raciste_4791000_3212.html, Jeune Afrique (31 juillet 2015) http://www.jeuneafrique.com/ mag/250227/culture/theatre-un-blanc-dans-la-peau-dothello/, Huffingtonpost (27 oct. 2015) http://www.huffingtonpost.fr/yasmine-modestine/othello-luc-mondy-

blackface_b_8388714.html

4. ille: dans le cadre de l'usage d'une langue non-sexiste, "ille" est un terme trans-sexe regroupant les pronoms masculins et féminins.

5. Le tableaux d'emploi élaboré en 1922 par Meyerhold, et où se trouvent associés, à différents types de rôles masculins et féminins, des profils physiques, vocaux et psychologiques très contraignants, permet de bien prendre la mesure des présupposés qui dictent les règles de ces structures de sens et modèles de représentation standard que sont les emplois (cf. Vsevolod Meyerhold, «L'emploi de l'acteur (1922) » in Ecrits sur le théâtre, tome 2 (dir. Picon-Vallin, nouvelle édition revue et augmentée), Lausanne, L'Âge d'homme, 2009).

6. Diomedes Gariazzo Lechini, «La distribution des rôles dans la tragédie athénienne », Agôn [En ligne], Distribution, rôles et processus de création : du point de vue des auteurs, (2015) $\mathrm{N}^{\circ} 7: \mathrm{La}$ Distribution, Dossiers, mis à jour le : 29/10/2015, URL : http://agon.ens-lyon.fr/index.php? $\mathrm{id}=3218$.

7. Céline Candiard, «Emplois comiques et répertoire moliéresque : enjeux dramaturgiques d'un principe de distribution ", Agôn [En ligne], (2015) No 7 : La Distribution, Dossiers, Distribution, rôles et processus de création : du point de vue des auteurs, mis à jour le : 12/10/2015, URL : http://agon.ens-lyon.fr/index.php?id=3179.

8. Stéphane Hervé, «La distribution comme phénomène pop: l'antiteater de Rainer Werner Fassbinder et la Needcompany de Jan Lauwers ", Agôn [En ligne], Distribution, rôles et processus de création : du point de vue des auteurs, (2015) $\mathrm{N}^{\circ} 7:$ La Distribution, Dossiers, mis à jour le : 29/10/2015, URL : http://agon.enslyon.fr/index.php?id=3183.

9. Esther Gouarné, « Wat gaan we vandaag spelen? À quoi joue-t-on aujourd'hui ? Les distributions organiques de la création collective néerlandophone. ", Agôn [En ligne], Distribution, rôles et processus 
de création : du point de vue des acteurs et des metteurs en scène, (2015) $\mathrm{N}^{\circ} 7:$ La Distribution, Dossiers, mis à jour le : 05/11/2015, URL : http://agon.ens-lyon.fr/index.php?id=3272.

10. Noëlle Renaude, «Ecrire, non pas pour des acteurs, mais pour des personnes ", Agôn [En ligne], Distribution, rôles et processus de création : du point de vue des auteurs, (2015) $\mathrm{N}^{\circ} 7: \mathrm{La}$ Distribution, Dossiers, mis à jour le :

11. /10/2015, URL : http://agon.ens-lyon.fr/index.php?id=3354.

12. Voir Jacques Rancière, La Haine de la démocratie, Paris, La Fabrique, 2005.

13. Le Collectif $X$, « «C'est assez bluffant la manière dont on s'attache à ces histoires de distribution! » (Partie 1: Le Soulier de Satin) », Agôn [En ligne], Distribution, rôles et processus de création: du point de vue des acteurs et des metteurs en scène, (2015) $\mathrm{N}^{\circ} 7:$ La Distribution, Dossiers, mis à jour le : 06/11/2015, URL : http://agon.enslyon.fr/index.php?id=3465

14. Violaine Chavanne, «Les «degrés zéro » de la distribution dans le théâtre de Jérôme Bel », Agôn [En ligne], Distribution, rôles et processus de création : du point de vue des acteurs et des metteurs en scène, (2015) $\mathrm{N}^{\circ} 7:$ La Distribution, Dossiers, mis à jour le : 26/10/2015, URL : http:// agon.ens-lyon.fr/index.php?id=3313.

15. Ismaël Jude, «Une distribution nomade », Agôn [En ligne], Distribution, rôles et processus de création: du point de vue des acteurs et des metteurs en scène, (2015) $\mathrm{N}^{\circ} 7:$ La Distribution, Dossiers, mis à jour le : 26/10/2015, URL : http://agon.ens-lyon.fr/index.php?id=3290.

16. Martin Crimp, Atteintes à sa vie, Paris, L'Arche, 2009.

17. Sur cette notion de "polytropie", voir: Guillaume Navaud, Persona. Le théâtre comme métaphore théorique de Socrate à Shakespeare, Genève : Droz, 2011, p. 249-286.

18. Matthieu Hocquemiller, «Processus de distribution dans/de la performance postpornographique », Agôn [En ligne], (2015) Nº 7 : La Distribution, Dossiers, Distribution, rôles et processus de création : du point de vue des acteurs et des metteurs en scène, mis à jour le: 12/10/2015, URL : http://agon.ens-lyon.fr/index.php?id=3262.

19. Camille Khoury, "Le travesti dans le théâtre du XIX ${ }^{\mathrm{e}}$ siècle: une distribution à contregenre ?», Agôn [En ligne], (2015) N 7 : La Distribution, Dossiers, Distribution, rôles et processus de création : du point de vue des acteurs et des metteurs en scène, mis à jour le : 05/11/2015, URL : http://agon.ens-lyon.fr/index.php?id=3448.

20. Marie Astier, «La distribution de comédien(ne)s en situation de handicap dans les spectacles de Philippe Adrien: enjeux éthiques, esthétiques et dramaturgiques ", Agôn [En ligne], Distribution, rôles et processus de création: du point de vue des acteurs et des metteurs en scène, (2015) $\mathrm{N}^{\circ} 7:$ La Distribution, Dossiers, mis à jour le :

21. /10/2015, URL : http://agon.ens-lyon.fr/index.php?id=3338.

22. Dorcy Rugamba, “Entretien”, publié le 2 février 2015, consulté le 31 octobre 2015, URL :

http://blogs.mediapart.fr/blog/amnesty-international/020215/entretien-avec-dorcy-rugamba-

dramaturge-rwandais ${ }^{21}$ Angela Davis, Femmes, race et classe, Paris, édition Des Femmes, 2013, p. 28.

23. Arnaud Maisetti, "Koltès - L'injouable du corps ", Agôn [En ligne], Distribution, rôles et processus de création : du point de vue des auteurs, (2015) $\mathrm{N}^{\circ} 7:$ La Distribution, Dossiers, mis à jour le : 27/10/2015, URL : http://agon.ens-lyon.fr/index.php?id=3334.

24. Voir le rapport 2015-2016 sur la parité Homme/Femme publié sur le site de l'association: http://www.hfidf.org/wp-content/uploads/SACD-Brochure-A5-2015-0820.pdf

25. Ce fut le cas par exemple d'une production montée par la Royal Shakespeare Compagny d'une pièce chinoise, The Orphan of Zhao, mise en scène par Gregory Doran au Swan Theatre à StratfordUpon-Avon en 2012-2013. La distribution s'est faite de manière complètement impensée en défaveur complète des acteur.trices d'origine asiatique, cantonnés aux rôles muets, secondaires, non individualisés ("deux chiens et une servante”...). Voir à ce titre Vanessa LEE, « "In Search of the Orphan" : Intercultural Theatre, Multi-Ethnic Casting, and the Representation of Chineseness on European and North American Stages. », Transtext(e)s Transcultures 跨文本跨文化 [En ligne], 7 
| 2012, mis en ligne le 02 décembre 2012, consulté le 05 novembre 2015. URL: http:// transtexts.revues.org/455

26. Cisgenre : terme qui décrit une identité de genre où la personne à une perception de son identité de genre identique à celle de son sexe de naissance.

27. Validiste: terme décrivant une société faite par et pour des personnes valides, où les personnes en situation de handicap sont marginalisées.

28. On pourra notamment se reporter à l'analyse de Peggy McIntosh (1988) sur le "privilège blanc": http://basseintensite.internetdown.org/IMG/pdf/privilegeblancmasculin.pdf; à Julia Serano (2008) sur le "privilège cissexuel": https://infokiosques.net/IMG/pdf/ le_privilege_cissexuel-28p-A4-fil.pdf ; à une variation "privilège masculin" sur le modèle de celui de Peggy McIntosch: http://www.as.utexas.edu/ cmcasey/diversity/male_privilege.pdf; synthèse transversale des différents privilèges

(des hommes, des hétérosexuel.le.s, des blanc.he.s, de classe, :

http://www.tcmfm.ca/fichiers_tcmfm/documents/Texte_de_r\%E9flexion_privil\%E8ges.pdf 28 Victor Hugo, Théâtre, Paris, Charpentier libraire éditeur, 1841, p. 313.

29. Aline Jaulin, «Exister à l'ère de la flexibilité chorégraphique : les gens d'Uterpan dans Avis d'audition", Agôn [En ligne], Dossiers, (2015) N 7 : La Distribution, Distribution et rapport de production, mis à jour le : 29/10/2015, URL : http://agon.ens-lyon.fr/index.php?id=3245.

30. Daniel Urrutiaguer, "Distributions et inégalités de valorisation dans le spectacle vivant ", Agôn [En ligne], Distribution et rapport de production, (2015) $\mathrm{N}^{\circ} 7:$ La Distribution, Dossiers, mis à jour le : 29/10/2015, URL : http://agon.ens-lyon.fr/index.php?id=3205.

31. C'est exactement le sens de l'acronyme STAN dans Tg STAN : Stop Thinking About Names (Tg étant mis pour « Toneelspelersgezelschap ", littéralement " compagnie de joueurs de théâtre »).

32. Marion Guyez, «La distribution sur les scènes circassiennes ", Agôn [En ligne], (2015) º 7 : La Distribution, Dossiers, Distribution et rapport de production, mis à jour le : 23/10/2015, URL : http://agon.enslyon.fr/index.php?id=3260.

33. Yves-Noël Genod, "Yves-Noël Genod, un théâtre de l'invitation ", Agôn [En ligne], Distribution et rapport de production, (2015) $\mathrm{N}^{\circ} 7$ : La Distribution, Dossiers, mis à jour le : 14/10/2015, URL : http://agon.enslyon.fr/index.php?id=3350.

34. Flore Augereau, "Conditions d'entrée et critères de sélection des formations françaises d'acteur : quels élèves pour quelles conceptions théâtrales? ", Agôn [En ligne], (2015) № 7 : La Distribution, Dossiers, À l'école de la distribution, mis à jour le: 12/10/2015, URL : http:// agon.ens-lyon.fr/index.php?id=3307.

35. Frédéric Plazy, «La vie d'un groupe à l'école : fabrique et dynamiques », Agôn [En ligne], À l'école de la distribution, (2015) $\mathrm{N}^{\circ} 7$ : La Distribution, Dossiers, mis à jour le :05/11/2015, URL : http://agon.enslyon.fr/index.php?id=3359.

36. Stanislas Nordey, « « Ouvrir le théâtre à tous » ", Agôn [En ligne], (2015) N 7 : La Distribution, Dossiers, À l'école de la distribution, mis à jour le : 05/11/2015, URL : http://agon.ens-lyon.fr/ index.php?id=3450.

37. Le Collectif $X$, « «C'est assez bluffant la manière dont on s'attache à ces histoires de distribution!» (Partie 2 : L'école) », Agôn [En ligne], À l'école de la distribution, (2015) N $7:$ La Distribution, Dossiers, mis à jour le: 06/11/2015, URL: http://agon.ens-lyon.fr/index.php? $\underline{\mathrm{id}=3475}$.

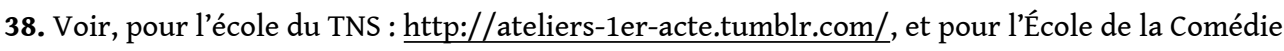
de Saint-Étienne le dispositif "égalité des chances" : http://www.lacomedie.fr/index.php/fr/lecole-de-la-comedie/egalite-des-chances 